\title{
Maps for the College Library
}

$\mathrm{M}$ APs should be an integral part of a college library, but relatively few librarians have considered them very important. This lack of interest results partly from the peculiar problems of handling and storing maps but even more from a lack of understanding of the nature and function of maps as reference materials. The increased demand for maps in educational work during and since World War II emphasizes the deficiency of map resources in libraries.

As reference and source material in a library, maps have two functions. First, facts about the earth's surface may be recorded on them. Second, and more important because it is an unique characteristic, they are a medium for expressing spacial relationships, especially distance, pattern outlines, and density distributions. The first of these functions may be performed by books also, but in many cases maps are still a more economical medium. A few lines on a map may record what several pages of text would require. In contrast, the second of these functions can be performed by books only in an extremely limited manner. Words may be sufficiently descriptive of a simple pattern, but a complex one in most cases is lost to all except the most imaginative reader. The pictorial aspect of a map expresses to the mind a multitude of spacial facts and relationships at a glance which could not otherwise be comprehended. A map is, therefore, an integral piece of source and reference material in a library.

Maps are used in colleges in three different ways to perform the functions described above: ( $\mathrm{I}$ ) in the classroom as wall maps and slides, (2) in the laboratory as tools, particularly for geography and geology, and (3) in the library as reference and source materials. The first two uses are wholly within the province of the teacher and classroom, and maps for these purposes should be kept independent from the library. The purpose of this article is to propose to the librarian a method of planning a map collection for the third of these uses within a college.

The reference and source map facilities required by college libraries obviously will vary greatly. The development of an adequate collection, however, can be and should be planned systematically through application of a few simple procedures and principles. The result will be a balanced collection which is tailored to the particular needs of the college and which can be enlarged in an orderly fashion.

\section{Determining Factors in Selection}

Four factors should be kept in mind when planning and selecting maps for any library: (I) the areas or parts of the world for which maps or coverage are desired, (2) the types of maps required, (3) the relative intensity of the coverage or scale over any particular area which is deemed necessary, and (4) the date of the material to be depicted. Any one or all four of these factors are limited by the material available. They may be used, however, in the preparation of an outline which will provide for purchases over a period of time.

The first of these factors, the geographic area, is more or less self-explanatory. Ob- 
viously one would want maps of the world, the hemispheres, the continents, and major parts of the continents. As the units of area become smaller, a problem of selection arises. For instance, a map of each of the forty-eight states would be desirable, but one of each of the more than three thousand counties might be beyond the scope desired. On the other hand, coverage might be obtained for all counties in the state in which the collection is located. This same reasoning can be applied to a continent or to any other given area. Maps of each of the political units in Africa might be of relatively little value, and, therefore, coverage could be provided by series of maps which cover continental parts made up of several political units. The choice of city maps for a collection provides another example of unit area selection. A representative program might include city maps for all places with more than $\mathrm{I}, 000,000$ population. Additional city plans for all places in Europe and the United States with more than 750,000 and 500,000 population respectively might supplement the world coverage. The basic collection could be supplemented further in various ways by addition of maps covering capital cities, important ports, historical cities, and nearby urban units. The first step is, therefore, the preparation of a list of unit areas for which maps are desired. It will be more valuable as a checklist if arranged systematically and on some regional basis. The larger land units should be broken down successively into smaller units, such as parts of continents, countries, and parts of countries. ${ }^{1}$ The extent of the

1 There are certain advantages in preparing one's own list with the help of an atlas. Several lists, how. ever, are available; see Samuel W. Boggs. "Library, Classification and Cataloging of Geographic Material." Annals of the Association of American Geographers 37:49-93, June 1939. Also: Samuel W. Boggs and Dorothy C. Lewis. The Classification and Cataloging of Maps and Atlases. New York, Special Libraries Association, 1945, p. 96-1r8. Also: Library of Congress. Classification Class G: Maps G 3I60-9999. Preliminary draft. Mimeographed pamphlet. Washington, Library of Congress, 1946, p. 5-47. breakdown will vary with the needs of the particular library. It will be governed by such considerations as the location of the school, the nature of the curriculum, and the types of requests received.

\section{Types Needed}

The second factor to consider in planning the collection is the types of maps needed. The great variety of maps available makes the selection process difficult if some classification scheme is not used as a guide. Maps may be divided into two broad groups, general and specialized. A general map is one that shows only such features as are common to most maps. A good illustration is the ordinary atlas map. A specialized map is one that emphasizes some particular feature or is designed for a particular need. Specialized maps may be subdivided into a number of major groups: physical geography, mathematical geography, political geography, economic geography, anthropologic or social geography, biogeography, and historical geography. These relatively comprehensive headings may be further subdivided several times into increasingly specialized units. As examples, the physical and economic geography groups may be subdivided partially as shown below. ${ }^{2}$ The breakdowns are not intended to be complete or equally subdivided, but are merely suggestive.

\section{Physical Geography}

Physiography, geomorphology

Relief

Physical maps, land forms

Physiographic regions, physiographic diagrams

Physiographic processes

Geology

Paleontology

Hydrography

Rivers, river systems, drainage basins

2 There are several classifications of maps which have been designed for cataloging purposes and which may be used as a basis for planning the specialized needs of a particular library; see Boggs, op. cit., p. 49-93; Boggs and Lewis, op. cit., p. $129-4 \mathrm{I}$; Library of Con. gress, op. cit., p. 48-58. 
Lakes

Oceanography

Submarine topography

Movements of sea water

Composition of sea water

Meteorology and climatology

Insolation

Air temperature

Air pressure

Atmospheric circulation

Atmospheric moisture: precipitation, humidity, fog, drought, etc.

Weather forecasting; weather maps

Climatology: climatic regions, climatic changes, etc.

\section{Economic Geography}

\section{Economic regions}

Natural resources and their utilization

Conservation of natural resources

Land utilization

Mineral resources and production

Agriculture

Agricultural regions

Agricultural methods

Crops and vegetable products

Cereals: wheat, rice, corn, etc.

Root crops

Hay and forage crops

Fruits and berries

Tree crops: coffee, tea, rubber, etc.

Animal resources and animal products

Transportation and communication

\section{Land transportation}

Water transportation

Communication by wire and wireless

If the groups above and the other major groups were broken down completely to a similar degree, a greater variety in types of maps would be obtained than needed by the average college. Such a detailed classification may be used as a checklist from which to select important types of maps which are desirable. In making the final selection several considerations and procedures should be kept in mind. First, the framework of unit areas chosen can be utilized as a qualifying factor for the extent of the specialized maps purchased. In other words, it is not necessary to order a specialized map of a given type for each of the unit areas to be represented in the collection. World or continental coverage in a single map sheet may provide sufficient pattern detail or distributional information. In a case where the unit area checklist emphasizes the importance of a given part of the world by the greater extent of the areal breakdown, it is likely that additional special maps covering smaller land areas than continents are desirable. As an example of this reasoning, soil maps of the following areas are suggested as suitable for more general reference requests: the world, Europe, North America, United States, ${ }^{3}$ and county soil maps for the state in which the library is located.

\section{The Curriculum}

A second consideration in the selection of specialized maps is the content and scope of the curriculum. If the school were an agricultural college, a set of all the published United States county soil maps would be appropriate in the foregoing illustration. Climatic and weather maps of various countries, market analysis and trade area maps, geologic maps, and urban planning maps would be consistent with courses in meteorology, marketing, geology, and city planning. The librarian cannot be expected to be conversant with the nature and scope of these and other highly specialized maps. He should seek advice and ask for specific recommendations from faculty members in various fields. The geographer with his more catholic interest in distributional problems should always be an able adviser, but even he cannot cope with advanced and specific problems of the geologist, as, for example, detailed mine plans and subsurface geologic maps of the Bolivian tin district.

The scope and technical character of spe-

${ }^{3}$ U.S. Department of Agriculture. Atlas of American Agriculture, Pt. III, Soils of the United States, by C. F. Marbut. Washington, Government Printing 
cialized map material is illustrated in the foregoing paragraphs. Unfortunately, faculty members are not always mindful of their own needs. The librarian, however, can insure to a certain extent the balance and the ability of the map collection to meet most requirements. Without hesitation he should obtain world, continental, and United States coverage, showing the pattern and distribution of the basic natural and cultural (man-made) features of the earth's surface. As a minimum, this would include maps of climate, earth materials (geologic, economic, minerals, and soils), water and biotic resources, population (including race, religion, and language), types of productive occupation (agriculture and manufacturing), transportation and communication facilities, and trade. Beyond these basic materials, he may provide a framework within which to purchase supplementary material by developing a generalized program of subject and areal content with appropriate faculty representatives.

\section{Intensity of Coverage}

The third consideration, the relative intensity of the coverage, is closely allied and intimately related to the first two factors. Intensity of coverage is expressed by scales, both by their variety and size. The amount of information which may be compressed into a map depends for the most part on its scale. It is evident, therefore, that the larger the scale, the more detailed is the information which may be shown. As the area becomes smaller and the size of the map sheet remains the same, the scale of the map naturally increases. Further increase in scale with a constant sheet size results in an increase in the number of sheets necessary to cover a given unit area. The key character of a map is thus closely related to its scale. When the scale is large the information shown is detailed and spe- cific, and the map is concrete. When the scale is small, the map loses its concrete character and becomes more or less abstract in form.

The scale factor, because of this bearing on the character and, therefore, the utility of a given map, should be used to modify and refine further the checklists of unit areas and subjects described previously. Maps may be divided into three broad groups according to scale: small, medium, and large. Small-scale maps are those whose fractional representation, or ratio between a given distance on the map and that same distance on the earth's surface, is small. For our purposes small-scale maps may be considered to have a scale smaller than I : I,O0o,ooo and large-scale maps larger than $1: 30,000$ with the intermediate scales representing the medium-scale maps. For areas and subjects determined to be relatively unimportant and for which requests are limited to a general character, smallscale maps will meet most requirements. Medium-scale maps fulfil the need for ( $\mathrm{I}$ ) greater areal detail in general coverage and (2) greater pattern and distributional information of phenomena depicted on specialized maps. Further control of the coverage intensity is possible through selection of several scales of increasing size within this group. Large-scale maps are essentially plans in the detail depicted, and their use is limited to such needs.

\section{Date}

The last factor to consider in designing the map program is the date of the material to be depicted. Except where the historical element is involved, the map of any area or particular subject with the latest date is desired. In time, these maps in turn will provide a record of the past. This raises the question as to when a map should be replaced by a new one or by a later edition. 
No standard rule can or should be applied. Each situation should be weighed on its own merits. Several principles, however, are helpful. First, maps which depict mainly natural features are subject to relatively little change. Maps more than fifty years in age may be satisfactory. Exceptions exist where new maps incorporate surveys which use improved mapping and cartographic methods, or are the result of additional information and advancement in a specialized field. Second, the rate of change in cultural features depicted varies considerably from region to region. Replacement of editions would be far less frequent for an area such as western Europe where roads, railroads, and other cultural patterns are relatively stabilized compared with parts of the United States. Therefore, replacement policies should be conditioned to the inherent characteristics of the area or type of material involved.

A word of caution is appropriate to the uninitiated concerning dates on maps. They are often misleading as compared to book dates because of differences in production techniques. The date given may be that of survey, compilation, revision, publication, reprint, or edition. Distinction among the above dates is subject to variable practice. In addition, commercial publishers, fearing their maps will be outdated too rapidly, commonly use coded dates. The date on a map, therefore, may not be indicative of the composite textual information depicted. It is well to remember that a reprint, which may be labeled as a new edition, normally involves only minor changes which can be made directly on press plates. Furthermore, the compilation date on one map may be later than that of a second one, but both maps may be based on source material of the same date. There is no simple solution, but experience and careful comparison of similar sheets will show some of the differences among leading map agencies. ${ }^{4}$

Planning the procurement of material where the historical element is involved is a more difficult problem. The high cost of old maps, in many cases without justification, limits their collection by many libraries. For this reason, clear objectives and a concise program should be formulated which meets the institution's needs before purchases are begun. The objective may be merely to show the record of cultural changes for a portion of the United States, before and after certain critical periods or at intervals of ten years. The latter is valuable to the historian in statistical work where minor civil divisions have changed through the years. On the other hand, the objective may be to show political development of an area or steps in the progress of cartography. Collecting of maps as museum pieces is not within the province of the average college library. An exception is the preservation of local map material which otherwise might be lost. ${ }^{5}$

\section{Reproductions}

Many of the historical needs of the college library can be fulfilled by reproductions. There has been an increasing number of facsimile reproductions both in single sheets and portfolios. Famous and important historical maps of the whole and parts of the world, Europe, and the United States have been reproduced both here and in Europe. There exist also several compilations of matérials into historical atlases which bridge gaps not covered by original maps and which can take the place of expensive and rare items. A study of the problem will help in the preparation of a sound program and

4 A detailed study of dating practices by several national surveys was made during the war by the Army Map Service, Corps of Engineers, U.S. Army. The information has not yet been published.

An outstanding example is that of county atlases. Although large numbers of them were published in the latter half of the nineteenth and early twentieth centuries, the mortality among them has been high, and librarians have given them little attention. 
will pay for itself many times in this field.

The program which has been outlined in the previous paragraphs will appear to the librarian familiar with books as too dogmatic and lacking in selective comparison. He will feel that such a program does not call for, or permit, sufficient evaluation of individual items. The answer lies in some of the inherent differences between maps and books. The great bulk of the maps published (large- and medium-scale topographic surveys) are issued by government agencies. The cost of field survey and publication makes their issuance prohibitive except by governments. Rarely are surveys duplicated. There is no competitive choice among items. In addition, topographic surveys are made according to certain scientific principles and are drafted according to standard specifications and symbols. They are designed to meet fixed values of accuracy, and variation in the end-products is strictly limited. If duplicating surveys exist, the selection among them may be, with some exceptions, more or less mechanical. It often will be decided by conditions other than those intrinsic to the map texts.

Compiled maps and many specialized maps (especially those on small scales), compared to field-surveyed maps, are subject to greater variation. The final products may express to a considerable extent the opinion and interpretation of the compiler. However, the degree to which this is possible is still less than among books in the same field. Similarly, the limitations in style are greater for maps than for books. A trick of map publishers illustrates these points concretely. Commercial firms commonly place an error of small importance within the map text to prevent direct copying of their product.

\section{Summary}

In summary, maps are a means of expressing certain facts which can be observed and measured. Their areal pattern and distribution are indicated by lines and conventionalized symbols with limited flexibility. As a medium of presenting ideas and information, they are less subject to personal judgment, delicate differences, and personal style than books. The librarian's first problem is to obtain professional help in outlining a program which satisfies the institution's requirements. The scope and content of the program can be stated in terms of the factors discussed: area, subject, scale, and date depicted. ${ }^{6}$ The librarian can make the necessary acquisitions within this framework. The problems of selection or choice among items available will be relatively few with this framework as a guide. His more serious problem will be the sources from which he can acquire maps which meet the specifications prescribed.

- A sample of such a program is illustrated by examples given for certain areas in a previous paper by the author. Specific titles need not be used as in these "Building a Collection of Maps." Bulletin of the American Library Association 30:206-15, April 1936, pt. 2. 